De Boeck, S., Tielens, P., Leterme, W., Van Hertem, D. (2013). Configurations and Earthing of HVDC Grids. Proc. IEEE PES GM 2013. IEEE Power \& Energy Society General Meeting. Vancouver, Canada, 21-25 July 2013 (pp. 1-5).

Digital Object Identifier: 10.1109/PESMG.2013.6672808

URL:

http://ieeexplore $\cdot$ ieee. org/stamp/stamp. jsp?tp=\&arnumber $=6672808$

(C) 2013 IEEE. Personal use of this material is permitted. Permission from IEEE must be obtained for all other users, including reprinting/ republishing this material for advertising or promotional purposes, creating new collective works for resale or redistribution to servers or lists, or reuse of any copyrighted components of this work in other works. 


\title{
Configurations and Earthing of HVDC Grids
}

\author{
Steven De Boeck, Graduate Student Member, IEEE, Pieter Tielens, Graduate Student Member, IEEE, \\ Willem Leterme, Graduate Student Member, IEEE and Dirk Van Hertem, Senior Member, IEEE
}

\begin{abstract}
HVDC grids are considered to be essential building blocks for the future upgrade of the existing AC power system and as a means to transport the expected massive amounts of renewable energy from remote sources to the load centers. HVDC systems exist for over 50 years, yet meshed DC grids do not exist so far. For point-to-point HVDC connections, there is a certain freedom in choosing the configuration and earthing scheme. For a grid, different converter arrangements and earthing schemes can be considered. The choices made will influence how the grid will look like, the components in the grid and their rating, the operating principles, the protection philosophy, the degree to which the grid is extensible and the overall reliability and inherent redundancy. Clearly, it will influence investment and operating costs as well.

This paper provides a qualitative overview of potential grid configurations for DC grids (symmetrical monopole, asymmetrical monopole, bipolar schemes, with and without metallic return and combined systems). The possible earthing options for a meshed HVDC grid are part of this discussion. The extensibility and reliability of the HVDC grid are specifically dealt with.
\end{abstract}

Index Terms-HVDC configuration, DC Earthing, Meshed DC grids, supergrid

\section{INTRODUCTION}

$\mathbf{I}$ $\mathrm{N}$ order to fundamentally upgrade the pan-European transmission system and to connect the foreseen large offshore wind farms, new transmission links are required.

ENTSO-E (European Network of Transmission System Operators for Electricity) expects that over $52300 \mathrm{~km}$ of new or upgraded transmission assets are needed in the coming decade in order to facilitate the internal energy market in Europe, to integrate the renewable energy sources and maintain a sufficiently high security of supply [1].

It is expected that due to the distances and required power ratings, a considerable part of these links will use HVDC instead of HVAC. Especially for submarine connections, the use of HVDC is necessary. In Europe, the construction of $12600 \mathrm{~km}$ of (mainly submarine) HVDC lines has already been planned in the next ten years [1]. When the HVDC links are connected and form a meshed DC grid, it can act as a new backbone grid of the European power system, also referred to as an overlay or supergrid [2]. The resulting system will form a hybrid AC-DC network. To achieve this supergrid, a transition from point-to-point connections towards a multi-terminal and meshed DC-grid is needed. To reach this goal, different choices regarding technology have to be made. This paper focuses on two of these aspects which are often disregarded: grid layout and earthing schemes.

S. De Boeck, P. Tielens, W. Leterme and D. Van Hertem are with the ELECTA research group, Electrical Engineering Department ESAT, Kasteelpark Arenberg 10, 3001 Heverlee, Belgium

Willem Leterme holds a Ph.D. fellowship from the FWO Flanders.

Contact: steven.deboeck@esat.kuleuven.be, pieter.tielens@esat.kuleuven. be, willem.leterme@esat.kuleuven.be, dirk.vanhertem@esat.kuleuven.be
The HVDC converters which is most suitable for DC grids is the VSC (voltage source converter) technology. Although CSC HVDC is a more mature technology and has still higher power ratings than VSC HVDC, the latter allows rather straightforward development of multi-terminal and meshed DC grids. Furthermore, VSC HVDC offers more controllability to AC grids through control of both active and reactive power [2], [3]. In this paper only VSC HVDC technology is considered.

Existing HVDC systems show a variety of configurations and earthing schemes, also for the relatively simple pointto-point HVDC connections. Systems with a monopolar or bipolar configuration exist, and for both there are systems with and without a metallic return. The DC system can be solidly earthed at one converter or both, or it can be ungrounded (high impedance earth) as is the case for most VSC HVDC schemes. For a grid however, not all DC grid layouts can be combined and the earthing scheme in one part of the grid influences the operation and protection of the entire system. Also the optimal number of earthing points, earthing principle and configuration needs yet to be determined.

In this paper an overview of the different HVDC grid configurations and earthing options is given. Their influence on the operation, extensibility and reliability of the DC grid is described. In section II, the different VSC HVDC grid configurations are described. The different earthing schemes for the configurations are discussed in section III. In section IV, a comparison between the extensibility, post-fault operation and earthing of these configurations in a meshed DC grid is made. Some practical considerations towards the choice of the earthing points are also given. Section V concludes.

\section{HVDC GRID CONFIGURATION}

Currently, all HVDC schemes are point-to-point systems, except for two multi-terminal schemes (Italy-Corsica-Sardinia and Quebec - New England interconnection). Nevertheless, it is expected that multi-terminal and meshed DC grids ${ }^{1}$ are built in the future. In this section, the possible configurations for both the point-to-point connections as DC grids are described. HVDC connections can be built up in a monopolar configuration or a bipolar configuration. Fig. 1 shows six different configurations. The symbol E, connected to each possible earthing point, refers to the different earthing options as shown in Fig. 2 and further elaborated upon in section III. To limit the number of options and because the use of earth return is often restricted due to interference or corrosion problems [4], systems which depend on earth return for their operation are not treated.

Figures 1(a) and 1(b) show two monopolar configurations: the asymmetrical and symmetrical monopolar scheme. In the

\footnotetext{
${ }^{1}$ Throughout the paper, the term DC grid is used as a general term encompassing both multi-terminal schemes and meshed HVDC grids, unless otherwise stated.
} 


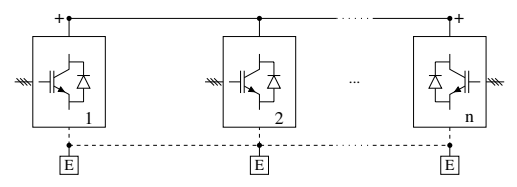

(a) Asymmetrical monopole system with earth return

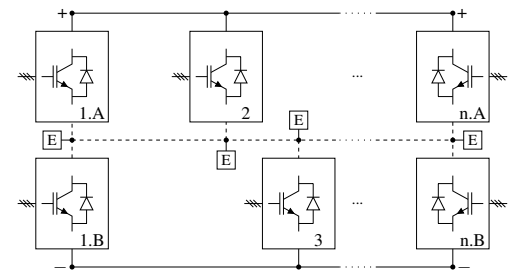

(d) Bipole system with metallic return and asymmetrical monopolar tappings

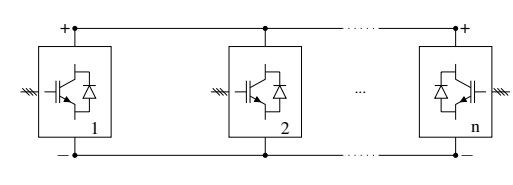

(b) Symmetrical monopole system

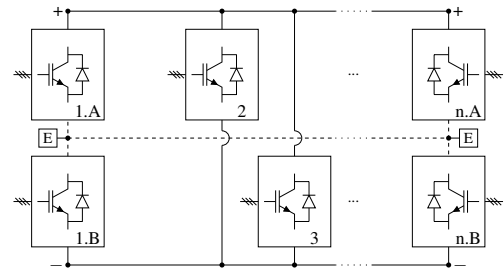

(e) Bipole system with metallic return and symmetrical monopolar tappings

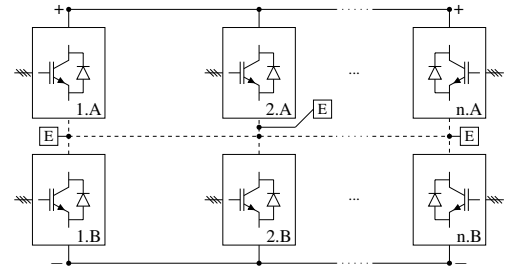

(c) Bipole system with metallic return

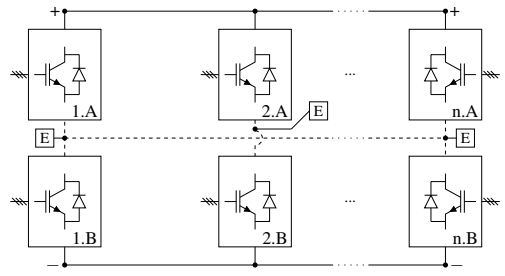

(f) Bipole system with metallic return and bipolar tappings with earth return

Fig. 1. Different VSC HVDC grid configurations (note that these configurations are in fact multi-terminal systems and not true DC grids.)

asymmetrical configuration, a low voltage conductor earthed at one or several terminals, is used as a return path. In the symmetrical configuration, the DC converters are connected to two high voltage conductors at opposite polarity. The earth reference can be provided with various methods, e.g. the connection of the DC capacitors' mid-point to the earth [5].

In a bipolar configuration (Figure 1(c)), two converters are connected in series at each terminal, one between the positive pole and the neutral point (the metallic return), the other one between the metallic return and the negative pole. In balanced operation, the voltages and currents in both poles are equal, and no current will flow through the metallic return. The bipolar configuration has the advantage that for a given rated pole voltage and rated current, the power that can be transmitted is double that of an equivalent asymmetric monopolar configuration with the same pole voltage and rated current. At the same time it offers the same power capability as a symmetric monopolar scheme, yet it offers the flexibility to operate at half the power when one pole is out, and therefore offers some inherent redundancy.

The three described configurations exist for point-to-point HVDC systems. The same topologies can also be used for DC grid, in the sense that they can be extended by using the same configurations in multiple points as already indicated by Fig. 1. However, it is also conceivable that the DC grid is built as a combination of multiple configurations. Such a system can be the consequence of the integration of different existing systems, or when for instance a smaller rated system is connected to a larger bipolar scheme. This smaller system might not need the full voltage rating, or the same reliability level. An example could be the connection of an offshore wind farm to an interconnection.

In Fig. 1(d) and 1(e), two configurations with monopole asymmetrical and symmetrical connections to a bipole system are shown. Another possible configuration is a bipolar grid where not all neutral points of the bipolar links are connected to a metallic return (Fig. 1(f)).

\section{EARTHING OF POINT-TO-POINT CONNECTIONS}

System earthing provides a means to keep the voltage to earth between predictable limits and allows the isolation of faulted equipment in fault situations [6]. Determining the system earthing is strongly coupled with the choice of system layout. Choosing the earthing scheme involves a trade-off between cost of insulation of components and the protection costs.

The different options to connect the neutral connection point with the earth are shown in Figure 2. The neutral point of the system can be connected to the earth (options I and II) or intentionally left unearthed (option III).

The first possibility is solid earthing of the system neutral point (switch connected to I). With this type of earthing, the voltage on each component during a short circuit is limited to the nominal voltage $U_{n}$. However, the steady-state fault current for pole-to-ground faults is limited solely by the conductor and earth resistance. To protect system components against overcurrents, the fault current must be interrupted as soon as possible, preferable before the steady-state current is reached. It is important to note that during a fault in a system with VSC HVDC (half bridge) converters and a solid earthed DC side, the fault current will be continuously fed from the AC side as the bypass diodes will take over the fault current after the IGBTs are blocked. Fast acting equipment (DC Breakers), working on a timescale of milliseconds are used to isolate the faulted component as soon as the fault is detected. For long lines, the use of protection schemes with communication may be impossible [7]. Even when solidly grounded, the earth impedance will be non-zero. For solidly earthed systems, this impedance must be kept as low as possible. The largest 
element of impedance in this situation is the impedance of the soil surrounding the earthing [8].

A second option is to earth the system neutral point through an impedance (option II in Figure 2). This impedance can in theory be a resistance, reactance or a capacitance. Resistance earthing can be used to limit the steady-state fault current in case of pole-to-ground faults. Low resistance earthing limits the voltage, while allowing a larger fault current. High resistance earthing limits the fault current, but causes a higher system voltage in ground fault situations. With reactance earthing the slope of the fault current can be decreased. However, the reactance will put a voltage between earth and the neutral connection point during the fault transient. This increases the voltage stress on components and raises the voltage on the healthy pole, which must be taken into account in the design phase. When using a capacitor coupling between earth and neutral point, the voltage excursions in case of pole-to-ground faults are limited. On the other hand, the grounding capacitor draws a large current immediately after the fault, imposing very stringent time constraints on the protection scheme.

Finally, the neutral connection point can be intentionally left unearthed or "high impedance earthed" (switch connected to III). In case of a single pole-to-ground fault, the theoretic fault current is zero. The voltage on the faulted pole is reduced to zero, while the voltage on the unfaulted pole jumps to a value of $U_{n}$ or $2 \cdot U_{n}$ for respectively the assymetric monopolar and bipolar scheme. For the asymmetric monopolar scheme, this type of earthing implies that both poles need to be insulated at nominal voltage and that one cannot use the "cheap" metallic return. For the bipolar scheme, the metallic return conductor needs to be insulated at nominal voltage, while the poles must be able to withstand twice the nominal voltage for the duration of a fault.

The main advantages of high impedance earthing are that there is no fault current and as such, the power supply on the faulted link can continue to operate in single-pole-to-ground fault situations. However, fault clearing action is required as a second fault on the other (healthy) pole or the metallic return conductor will form a double-pole-to-ground fault, resulting in very high currents. Time constraints for the acting equipment can be less stringent for this type of earthing. Guaranteeing selectivity by correct fault location can form a bigger problem in a highly meshed grid. This type of earthing is used in existing VSC HVDC systems, where fault acting equipment exists of the AC protection system, and the entire (point-topoint) DC link is disconnected.

When the system neutral connection point is left unearthed, the system is earthed through stray capacitances in the system, especially when using cable systems. As there is no imposed DC voltage reference, the steady-state DC voltage will be floating corresponding to leakage currents in the system [9]. A last option for the earthing might be the use of an active device, which switches the earthing connection depending on the state of the system. Such a system could be used to move from impedance to solid earthing.

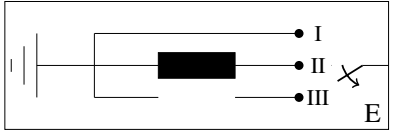

Fig. 2. Earthing options

\section{MESHED GRID CONSIDERATIONS}

In this section, the extensibility, reliability and a number of other issues related to the earthing and operation of the different HVDC grid implementations and their configurations are discussed. As the power system is in continuous development, it must be extensible to accommodate the (uncertain) future loads and generation. These extensions could be an additional mesh in the DC grid to increase the capacity of the system, a new connection to an AC system or a tapping to connect an offshore wind farm. A second driver for the grid development is the reliability of the HVDC grid. The perceived reliability with HVDC grids depends on different aspects: topology, configuration, type of contingencies taken into consideration, protection being used and type of earthing. It is realistic that a comparable or even higher reliability level than that of the AC system is aimed for.

\section{A. Extensibility}

1) Bipolar grid: A bipolar HVDC configuration with metallic return has the advantage of providing an inherent partial redundancy. Furthermore, it is a system that offers considerable flexibility towards extension. Clearly, it can be extended using bipolar systems, with or without metallic return. In the case that the connection is made without the metallic return and the bipolar tapping is not earthed, this is similar to a symmetric monopole connection on a bipolar grid (Fig. 1(e)).

Another possible extension is an asymmetric monopolar connection or tapping. Such a connection is made between the metallic or earth return and a pole (Fig. 1(d)). Unless there is an asymmetric tapping of the same rating at the same location to the other pole, which is operated identically, this results in an unbalanced operation of the HVDC grid and current will flow though the metallic or earth return. This unbalanced DC grid operation will propagate throughout the system causing different voltages and currents in the positive and negative pole as well as on the metallic return. Such an operation might have a significant effect on the required control systems of the converters connected to such a grid.

2) Symmetric monopolar grid: The symmetric monopolar grid can be extended with bipolar grid parts that offer a higher flexibility towards power ratings. The monopole then fixes the positive and negative voltage of the grid. Asymmetric extensions on a symmetric monopolar grid are not possible, as the ground of the symmetric monopole is not directly available.

3) Asymmetric monopolar grid: The asymmetric monopolar grid can only be expanded by other asymmetric monopoles or by a connection to one pole of a bipolar configuration. The asymmetric monopole in itself can be extended quite easily to a bipolar scheme. 


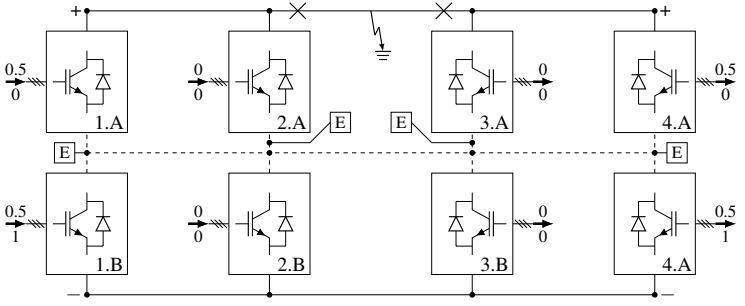

(a) Central approach

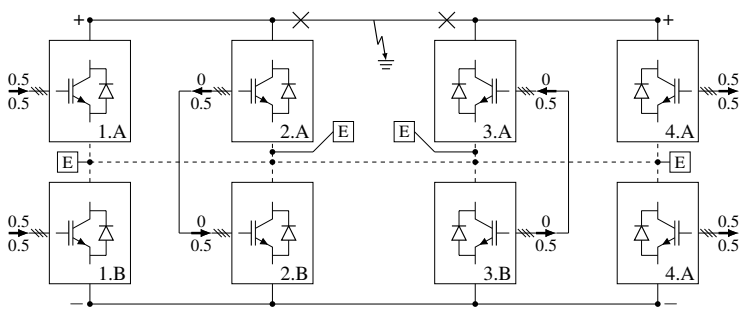

(b) Local approach

Fig. 3. Fault on pole of bipolar multi-terminal system. The rating of the system is $2 \mathrm{pu}$ ( $1 \mathrm{pu}$ for each pole). The situation before the fault is given above the arrow, the situation after the fault below. The section between the crosses is removed after the fault.

As described above the bipolar grid configuration offers the highest flexibility.

\section{B. Post-fault operation}

If we consider only a single HVDC configuration for a given grid, then there are three possibilities: full bipolar, full asymmetric monopolar and full symmetric monopolar. Depending on the configuration and the fault, a different postfault operation occurs.

1) Pole-to-ground fault or converter fault: In the case there is a fault on one of the poles of a symmetric monopole or on the pole of an asymmetric monopole, this will result in removing of this section of the grid. The original flow through this section will have to be redistributed over a parallel path.

A fault on a pole of a grounded bipolar grid requires the immediate removal of the fault by disconnecting that section from the grid. This results in switching to asymmetric monopolar operation for a part of the grid. The same is valid when a single converter of a bipolar configuration is removed. Initially the flows in the grid will be redistributed. This can in some cases lead to temporary overloads. The power which was originally carried by the faulted pole cannot be simply shifted to the healthy pole, even if there is still sufficient spare capacity on the healthy pole, without any changes in the HVDC control. The problem of the overloads and the shift of the power towards the healthy pole can be solved by two different approaches: a local or a central redispatch of the DC converter settings. Both approaches can be explained using the simple multi-terminal system shown in Fig. 3.

In the central approach, a central DC grid dispatch optimizes the infeeds and withdrawals from the DC grid, resulting in new set-points for the different DC converters. The problem can also be solved locally, by changing the set-point of the converters closest to the faulted section. The flow of the converter connected to the faulted pole, will be redirected to the converter connected to the healthy pole. This results in an AC flow from one converter to the other. In this case, one of the converters of a single bipole might be operate as a rectifier while the other operates as an inverter. In both the central and local case this results into an asymmetric redispatch and grid operation, as both poles will not be equally loaded. The change of set points will not occur instantaneous. The time needed depends on the implemented redispatch option: from some milliseconds in case of an automatic local action, to some seconds with a centralized automatic action using a state estimation or if a manual redispatch or human interaction is needed it might take up to a number of minutes.

A fault on a bipolar system with high impedance grounding will cause a direct earth connection, transforming the system into an earthed system. This system will not experience extreme fault currents, yet the pole-to-ground voltages throughout the DC grid will change, potentially causing any problems with insulation. Furthermore, a second fault, also remote from the first fault, might cause a large fault current for which the system is not designed. Such a double fault might require to disable the entire section between the two fault locations.

2) Fault on metallic return: A fault on the metallic return of an earthed bipolar system does not directly result in a high current, or the interruption of the flow through this section. If the DC grid is operated in an asymmetric mode, a current flows through the metallic return before the fault. After the fault these flows might be influenced. If the fault is on one of the asymmetric connections, this might cause the loss of that connection unless an earth return is used. Even when no asymmetric connections are used, the bipolar system will enter a less secure state of operation. The reason is that subsequent to a fault on one of the poles, the complete section will have to be removed temporarily.

A fault on the metallic return of an asymmetric monopole will immediately cause part of the current to flow through the earth. The division of the current between the earth and the metallic return depends on the ratio of the resistances of the earth and the metallic return.

In case of an ungrounded system, a fault on the metallic return will transfer the system into a grounded system, however, this might go unnoticed at first. In any case, a fault in the metallic return needs to be detected and cleared. In such a case, the system might be operated as a system without a metallic return in part of the meshed DC grid (comparable to the system with a symmetrical monopole as extension).

3) Fault on AC side: A fault on the AC side can always result in the loss of the converters connected to this $\mathrm{AC}$ node and thus the loss of a DC node.

4) Pole-to-Pole fault: Although unlikely, specifically in the case of a DC grid solely using cables, a pole-to-pole fault can occur.

\section{Earthing and operation}

Beside the choice of the earthing type (type I, II or III of Figure 2), some other considerations for earthing of a meshed 
DC grid have to be made. Specifically the number and location of earthing points has to be determined.

A first option is to ground the grid solidly at one single point. Consider Fig. 1(c), with one point solidly earthed and the others are left unearthed. If a fault occurs on a pole of this scheme, the total fault current will flow directly from the fault location to the solidly earthed neutral point. The voltage on the neutral points of the unearthed converters increases due to the current flowing through the metallic return conductors. In the bipolar scheme, this will cause a current to flow in the other pole resulting from the unbalanced conditions. This can lead to selectivity and sensitivity problems for fault acting equipment if communication between relays is impossible. The voltage at the non-earthed neutral point located far from the ground might rise to values which are unacceptable. The large fault currents would also cause a considerable rise in the ground potential near the earthing point. Another effect is the return path of the current. In AC systems, such fault current flows follow a path which roughly runs along the conductors because of inductive coupling. The absence of this inductive coupling in DC leads to current paths in the earth that do not necessarily lie along the conductors. This can cause damage on pipelines and other metallic infrastructure along these paths due to corrosion.

Alternatively, the system can be solidly grounded at multiple converter substations. Consider again Figure 1(c), with each neutral point solidly grounded. For this grounding scheme, stray currents will flow through the earth, even in steady-state when the system is operated in unbalanced conditions. These currents affect the ground potential distribution. Stray currents are also subject to environmental constraints. Furthermore, DC currents can flow via the $\mathrm{AC}$ earth connections through nearby AC system. This DC current can cause saturation and even damaging effects on the AC transformers. As a consequence, problems related to power quality and safety system malfunctioning arise in the AC grid [10]. As an advantage, during ground fault situation, the fault current will be divided among the different neutral points according to the earth impedance. Voltage differences between the neutral points in the DC system are lower than those in a scheme with a single earth.

In realistic DC grid situations, multiple earth connection points are needed. For the first case, one connection point must be solidly grounded while others can be used as backup and only connected when needed. In the second case, earth connection points have to be chosen in an intelligent manner. The first consideration is limiting the voltage of other neutral points in the DC system due to fault currents. As a supergrid covers a large area, geographic issues come into consideration. Earth conductivity between remote neutral points can be small due to discontinuities of earth layers as a consequence of different tectonic plates. One option might be to solidly earth the system at a single point, while earthing the system through an impedance at other locations with a bypass switch.

A third option is high impedance earthing of the whole system, resulting in a floating voltage system. While this is also used in current VSC HVDC systems, it is not likely that such a system will be used for (large) DC grids. Firstly, the cable insulation has to be dimensioned to accommodate a voltage which is up to the double of the nominal voltage. This will add a significant cost for the realisation of the these schemes. Secondly, the current protection system operates by clearing the AC side of the converters. For larger systems, such an outage is not likely to be acceptable.

\section{CONCLUSion}

This paper gives an overview of grid configurations and earthing possibilities for DC grids. The grid configuration has a considerable effect on the operation, extensibility of the system and the overall reliability of energy provision. This influence is pronounced and not yet studied so far. In a qualitative assessment, the bipolar grid configuration turns out to be the configuration that matches these technical criteria. A strongly linked topic is the earthing scheme of the DC grid. The type of earthing, location and number of earthing points affects the protection scheme, the cost of components and grid operations. Additionally, effects on metallic and electrical structure and environmental aspects along earth current paths need to be considered. Finally, it is important to note that the protection scheme has a considerable impact on the fault currents, the interactions between the DC and AC grid and the flow distribution.

\section{REFERENCES}

[1] ENTSO-E, “10-year network development plan 2012," ENTSO-E, Tech Rep., 2012.

[2] D. Van Hertem and M. Ghandhari, "Multi-terminal VSC HVDC for the European supergrid: Obstacles," Renewable and Sustainable Energy Reviews, vol. 14, no. 9, pp. 3156 - 3163, 2010.

[3] ENTSO-E, "Offshore transmission technology," ENTSO-E, Tech. Rep., 2011.

[4] J. Arrillaga, Y. Liu, and N. Watson, Flexible Power Transmission: The HVDC Options. Wiley, 2007.

[5] Working Group B4-52, "Hvdc grid feasibility study," Cigré, Tech. Rep., 2012.

[6] IEEE IAS: Power System Technologies Committee, IEEE recommended practice for grounding of industrial and commercial power systems, ser. IEEE Green Book. IEEE, 2007.

[7] D. Van Hertem, M. Ghandhari, J. B. Curis, O. Despouys, and A. Marzin, "Protection requirements for a multi-terminal meshed dc grid," in Cigré International Symposium The Electric Power System of The Future : Integrating supergrids and microgrids, Sep. 2011.

[8] T. Gönen, Electrical Power Transmission System Engineering Analysis and Design: Analysis and Design, 2nd Edition. CRC Press, 2009.

[9] B. Jacobson and J. Walker, "Grounding considerations for dc and mixed DC and AC power systems," Naval Engineers Journal, vol. 119, no. 2, pp. 49-62, 2007.

[10] B. Zhang, J. Zhao, R. Zeng, and J. He, "Numerical analysis of DC current distribution in AC power system near HVDC system," Power Delivery, IEEE Transactions on, vol. 23, no. 2, pp. 960 -965, apr. 2008. 